\title{
ZUR DATIERUNG DER IN DEN VECKINCHUSENSCHEN HANDELSBÜCHERN GENANNTEN MESSEN
}

Die Anmerkungen beziehen sich in der Hauptsache auf die genauere Feststellung der im Text genannten Daten. Im großen und ganzen sind die Posten in den Büchern Af 1 und Af 6 in chronologischer Ordnung eingetragen; die Datierung ist aber sehr unzulänglich. Die Jahresangaben sind äußerst spärlich, was einer genauen Feststellung der Reihenfolge und der Daten der Geschäfte oft unüberwindliche Schwierigkeiten bereitet, besonders wenn das Datum an einen beweglichen Fest- oder Messetag oder an einen diesen Daten vorangehenden oder nachfolgenden Wochentag gebunden ist. Bei der Auswertung der Quelle ist es aber oft notwendig, das Datum genau festzustellen, um sich Möglichkeiten zu verschaffen, aus den Angaben wichtige Rückschlüsse zu ziehen, z. B. über die Geschwindigkeit des Umsatzes oder über die Kreditbedingungen. Es wird z. B. berichtet, daß eine am 14. März angekommene Warenpartie auf der Ypernmesse verkauft wurde. Im Jahre 1404 begann die Messe am Aschermittwoch, dem 12. Februar; im Jahre 1405 am 4. März; im Jahre 1406 am 24. Februar; im Jahre 1407 am 9. Februar. Das heißt also, daß, wenn die Jahre 1405 oder 1406 gemeint sind, die Waren fast sofort abgestoßen werden konnten, in den Jahren 1404 oder 1407 aber einige Monate bis zur Pfingstmesse - im Lager liegen blieben. Ein anderes Beispiel: Ein Kreditgeschäft wurde am 15. April abgeschlossen und als Zahlungstermin die "nächstkommende Brüggermesse" verabredet. In den Jahren 1411 oder 1413 hätte das eine drei- bis vierwöchige, für die Jahre 1410 oder 1412 eine einjährige Kreditgewährung bedeutet.

Da die Messen sehr oft als Zahlungstermine sowie auch als Ankaufs-, Verkaufs- und Zahlungsorte in den Quellen erscheinen, müssen ihre Daten besonders genau festgestellt werden.

Leider aber sind nicht alle Fragen der Datierung flandrischer und brabantischer Messen vollständig geklärt, auch nicht deren innere Zeiteinteilung. Die im wissenschaftlichen Gebrauch benutzten Quellenangaben sind sehr dürftig, und ihre Auswertung in den einschlägigen und von uns benutzten Arbeiten ist nicht immer geglückt. Als Beispiel kann man die unterschiedliche Datierung der Brügger Messe anführen. Es werden folgende Daten angegeben: 
1) „1. Mai“ (Nirrnheim) ${ }^{1}$

2) "zweiter Tag nach der Osterwoche" (Huvelin) ${ }^{2}$

3) „erster Sonntag nach Ostern" (Stein) ${ }^{3}$

4) "Zweiter Tag nach der Osterwoche" (Des Marez)4

5) Montag nach „Osterschluß (Closes Pasques)“ (Poignant) 5

6) „dritter Montag nach Ostern“ (Laurent) 6

7) ,23. April“ (van Houtte) 7

Die dem Leser hier als Hilfsmittel vorgelegten Termintabellen ${ }^{8}$ beziehen sich auf die wichtigsten in den Veckinchusenschen Handelsbüchern erwähnten Messen: die von Antwerpen, Brügge, Kortryk (Courtrai), Torrolt und Ypern sowie ferner die von St. Denis (Lendit) und Frankfurt am Main. Sie umfassen nur das erste Viertel des 15. Jh. - die Jahre der Handelstätigkeit des Hildebrand Veckinchusen. Absolute Genauigkeit kann man von ihnen nicht verlangen, denn wegen der Dürftigkeit der Quellen ist beispielsweise über die Daten der flandrischen und brabantischen Messen für die Jahre von 1400 bis 1425 gar nichts bekannt: Die nächstfrühere Angabe hierüber stammt aus dem Jahre 1344, die nächstspätere von 1428.

Noch spätere Angaben - sie datieren aus den Jahren 1452 bis 1532 - sollen hier unberücksichtigt bleiben. Zum ersten nämlich widersprechen sie öfter den früheren Angaben und türmen damit weitere Schwierigkeiten auf; zum anderen aber sind sie ohnehin wegen ihres zeitlichen Abstandes für unsere

1 Das Handlungsbuch Vickos van Geldersen. Bearb. v. Hans NirRnHeIm. Hamburg u. Leipzig 1895. Auf S. XXXIX beruft sich N. auf S. Gilliodts van Severen, Inventaire des chartes de la ville de Bruges. Table analytique p. Edw. Gaillard, S. 191.

2 P. Hoverin, Essai historique sur le droit des marchés et des foires. Paris 1897 , S. 262. H. stützt sich auf einen Aufsatz von Gaillard, der von Des Marez als „trop inexacte" beurteilt wird.

Hansisches Urkundenbuch, 8, S. 113, Anm. 2, auf Grund von Gilliodrs vaN

3 Severen, Inventaire, 5, S. 358.

Guillaume des Marez, La lettre de foire a Ypres au XIII siècle. Bruxelles 1901,

${ }_{4}^{4}$ S. 82-84 u. 86. Mit Quellen belegt und richtig, obwohl wir mit seinen Auslegungen nicht immer einverstanden sind.

5 Das Buch von Poignant, La foire de Lille, Bruxelles 1932, blieb mir unzugänglich, aber aus den mir durch Professor J. van Houtte, Löwen, freundlicherweise übersandten Exzerpten erkenne ich, daß die Arbeit mit Ausnahme einiger auf die Liller Messen bezüglicher, keine neuen und mir unbekannten Quellenangaben enthält.

${ }^{6}$ Henri Laurent, Un grand commerce d'exportation. Paris 1935, S. 39. Das von L. angeführte Datum ist eine unverständliche Abweichung von Des Marez, dessen Angaben L. als die besten und zuverlässigsten schätzt.

7 van Hodtte, La Foire. Bruxelles 1953.

${ }^{8}$ Die Messezeit teilt sich gewöhnlich in vier Abschnitte: Eingang (Entrée), Schautage (Jours de monstre), Zahlungstage (Jours de payment) und Abfahrt (Jours de sortie). 
Zwecke ohne großen Belang. Und letzten Endes scheinen sie auch immer noch zu karg, um die Möglichkeit für sichere Rückschlüsse zu bieten.

Über die Brügger und Antwerpener Messen, welche in den Veckinchusenschen Büchern den bedeutendsten Platz einnehmen, sind noch einige Bemerkungen vonnöten.

Unter den oben angeführten sieben Aussagen über den Eröffnungstag der Brügger Messe gibt es vier, welche ihn auf den Montag nach Quasimodo festlegen $(2,3,4,5)$.

Abweichend hiervon geben Nirrnheim (1) und van Houtte (7) feste Daten an, nämlich den 1. Mai beziehungswêise den 23. April. Diese Behauptungen, die einen unkundigen Leser zu dem irrigen Schluß führen könnten, der Anfang der Messe sei wirklich an ein solches fixes Datum gebunden, lassen sich leicht erklären durch den Umstand, daß beide Verfasser den ständigen Wechsel des Osterdatums in keiner Weise berücksichtigt haben.9 Ihre Daten sind allein von den Messeterminen eines Jahres abgeleitet: Sie stimmen wohl dann, wenn wie im Jahre 1413 der Ostersonntag auf den 23. April (bzw. wie 1403 auf den 15. April) fällt, aber auch nur dann. Laurent schließlich stützt seine Behauptung wahrscheinlich auf eine sehr späte und nicht ganz eindeutige Quelle aus dem Jahre $1532^{10}$, die möglicherweise eine Wiederholung des Privilegs Herzog Philipps von 1452 ist ${ }^{11}$; er läßt jedoch alle früheren von Des Marez ausgewerteten Quellen ganz unbeachtet. In der genannten Urkunde von 1452 aber handelt es sich um eine von Philipp vorgenommene Veränderung der Messetage; über den früheren Zustand gibt es hier keine Aussagen.

Alle bekannten Quellen des 13. und 14. Jh. geben als Eröffnungstag der Messe nun wirklich den Montag nach Quasimodo an. So heißt es in einem Privileg des Grafen Balduin vom Jahre 1200: „feria secunda post octavas Pasche"12, und eine spätere Urkunde aus dem Jahre 1344 sagt sehr klar: „Li fiest à Bruges entre le lundi après Closes Pasques" 13 .

Von großer Wichtigkeit sind die Schautage (Monstre), deren Anfang die Dauer der Entrée und deren Ende das Datum der Zahlungstage bestimmte. Teilweise, wie z. B. in den Liller Quellen 14, werden sie sogar als Anfang der Messe betrachtet. Dieselbe Anschauung findet sich nach Des Marez auch in der Notiz des Jahres $1532^{15}$.

${ }^{9}$ Im Gegensatz zu Des Marez, a. a. O., S. 86, Tabelle; dieser mit der ausdrücklichen Bemerkung: „en supposant que Pâques tombe le 15 Avril“.

10 Eine handschriftliche Notiz vom Jahre 1532 auf einem Manuskript der Chronik des Olivier de Dixmude in der Bibliothek von Ypern; zitiert boi Des Marez, ebenda, S. 79, Anm. 2.

11 Der Text ebenda, S. 85, Anm. 1.

12 L. Warnkoznia, Flandrische Staats- und Rechtsgeschichte, Bd. II, S. 91; zitiert bei Des Marez, a. a. O., S. $82 \mathrm{f}$.

${ }^{13}$ G. Espinas, Documents relatifs à la draperie de Valenciennes, Lille et Paris 1931, nr. 450, S. 305.

14 Poignant, a. a. O., S. $89 . \quad$ 15 Des Marez, a. a. O., S. 79. 
Für Brügge haben wir über das Datum der Schautage eine genaue Angabe in der obengenannten Urkunde vom Jahre 1344. Es heißt nämlich im Anschluß an deren eben zitierten Text: „Et li premiers jours de monstre des dras est $a$ dies de ce lundi en 15 jours" 16.

Die Schautage fingen somit am Montag nach Jubilate an, und die Entrée dauerte 15 Tage. Der gleiche Termin wird generell für alle flandrischen Messen in einer viel früheren "Coutume de la ville de Bruge“ aus dem Jahre 1295 angegeben, welche auch die Dauer der Schau- und Zahlungstage aufführt: „Cascune des festes a quinze jours de sa entrée dusques au jour de monstre et trois jours de monstre et quatre jours de paiements après les jours de monstre. Coutume de la ville de Bruges 1295"17.

Die Entréedauer konnte jedoch auch kürzer sein. Des Marez ist der Meinung, daß eine achttägige Entrée die Regel war ${ }^{18}$. Diese Annahme scheint uns aber insbesondere für Brügge nicht stichhaltig zu sein, und sie kann auch zu einer fehlerhaften Datierung der Messeneröffnung führen 19.

Für einen fast 300 Jahre währenden Zeitabschnitt, nämlich von 1296 bis 1566, werden in der Literatur nur drei Quellenstellen angeführt, die von einer achttägigen Dauer der Entrée sprechen. Zwei davon, aus den Jahren 1296 und 1428, beziehen sich auf Lille (Ryssel), die dritte auf Messine (Meisen) ${ }^{20}$.

Für Lille haben wir andererseits einen sehr genauen Text des 14. Jh., in dem als Eröffnungstag der Messe der 15. August und als der erste Schautag (monstre des dras) der 27. August angezeigt sind ${ }^{21}$. Also dauert die Entrée hier unz weifelhaft 12 Tage. 22

Es sollen noch einige Bemerkungen über die Antwerpener Messen folgen, die in den Veckinchusenschen Handelsbüchern oft als Zahlungstermine oder als Zahlungs-, Ankaufs- und Verkaufsorte erscheinen.

In Antwerpen wurden im 15. Jh. zweimal im Jahre Messen abgehalten: die Frühjahrs- oder Pfingstmesse und im Herbst die „Bamysse“. Die Pfingstmesse

16 Espinas, a. a. O., nr. 450, S. 305.

17 Zitiert bei Des Marez, a. a. O., S. 84 .

18 Ebenda, S. 83: ,abstraction faite des huits jours d'entrée", Brügge, S. 80: „huit jours d'entrée“; Thourout, S. 85, Anm. 1 : „huit jours qui precedent l'ouverture (Thourout)".

19 Z. B. ebenda, S. 80, Anm. 3: Eröffnungstag der Thouroutmesse 3. Juli.

${ }^{20}$ Lille: "Octo dies ante inchoationem" (Geleitbrief von Philipp dem Schönen aus dem Jahre 1296; zitiert bei Des Marez, a. a. O., S. 84, Anm. 3). Lille: die MesseSchautage soll man ,tenir les 27,28 et 29 jours d'aoust avec bon, seur et loial saufconduit, huit jours avant et huit jours après ledit 29-e jour d'aoust" (Urkunde von Philipp dem Guten aus dem Jahre 1428; Poignant, a. a. O., S. 88 und 177). Messine: „trois jours de monstre et la franchise huicts jours devant" (Brief Philipps II. von Spanien aus dem Jahre 1566; zitiert bei Des MAREz, a. a. O., S. 82.

21 Zitiert bei Des Marez, a. a. O., S. 85, Anm. 1.

22 Privileg der Maria von Burgund aus dem Jahre 1477 : ,net langher dan drie toghedaghen naer dhoude costume" (vgl, Des MAREz, a. a. O., S. 85). 
begann 15 Tage vor Pfingsten und dauerte vier Wochen.23 Da die Antwerpener Herbstmesse in den Quellen „Bamysse“, die Messe des hl. Bavo, dessen Festtag auf den 1. Oktober fällt, genannt wird, hat man dieses Datum manchmal irrtümlich als den Eröffnungstag der Messe angenommen. ${ }^{24}$ Tatsächlich aber hat der Bavo-Tag weder zum Anfang noch zum Abschluß der Messe eine Beziehung.

Im 15. Jh. findet die „Entrée“ am zweiten Sonntag nach Mitte August 25 statt, also vom 23. bis 29. August 26. Die Messe dauert vier Wochen, und dementsprechend fällt ihr Schluß in die Tage vom 19. bis 25. September. Die Messe wird also in einigen Jahren schon elf Tage vor dem Bavo-Tage geschlossen.

Man findet in den Hanse-Akten nicht immer eine Bestätigung dieser Daten. Das Hansekontor zu Brügge pflegte für die Messezeit nach Antwerpen überzusiedeln. Das spiegelt sich auch in den Unterschriften der vom Hansekontor während der Messezeit ausgehenden Akten wider: „Alderlude des gemenen coopmans van der Duytschen Hanse uppe dese tyd to Andorp in Brabant in vrye markt wesende".27 Die Daten dieser Akten stimmen - was die Pfingstmesse betrifft - mit sehr wenigen Ausnahmen mit den Messedaten des entsprechenden Jahres überein, die auf die Bavomesse bezüglichen aber nur in zwei von fünf Fällen, wie aus der folgenden Tabelle ersichtlich ist. ${ }^{28}$ Dürfen wir aber daraus schließen, daß die Bamysse nicht 4 Wochen $=28$ Tage, sondern 1 Monat $=31$ Tage dauerte?

\begin{tabular}{|c|c|c|c|c|}
\hline \multicolumn{5}{|c|}{ Pfingstmesse } \\
\hline Quelle & Jahr & Pfingsten & Messezeit & $\begin{array}{c}\text { Datum } \\
\text { der Urkunde }\end{array}$ \\
\hline \multicolumn{5}{|l|}{$\overline{\text { HUB } 8}$} \\
\hline nr. 60 & 1451 & 13. Juni & 30. Mai-30. Juni & 29. Juni \\
\hline 466 & 1456 & 16. Mai & 2. Mai- 2. Juni & 16. Mai \\
\hline 583 & 1457 & 5. Juni & 22. Mai-22. Juni & 13. Juni \\
\hline 584 & 1457 & 5. Juni & 22. Mai-22. Juni & 14. Juni \\
\hline 591 & 1457 & 5. Juni & 22. Mai-22. Juni & 23. Juni \\
\hline $93 \tilde{3}$ & 1460 & 1. Juni & 18. Mai-18. Juni & 22. Juni \\
\hline
\end{tabular}

${ }^{23}$ VAN Hovtte, La Foire, a.a. O., S. 189 ; dagegen Huvelin, Essai historique, a. a. O., S. 264: Montag vor Pfingsten, also eine Woche später.

24 Z. B. in der Cambridge Economic History, Bd. 3, S. 150.

${ }^{25}$ Van Houtte, La Foire, a.a. O., S. 189. Laut dankenswerter Mitteilung von Prof. van Houtte stützen sich diese allgemein angenommenen Daten auf den Text des mir unzugänglichen Antwerpener Stadtrechts (Consuetudines Impresse 1582. Neudruck: Coutumes de la ville d'Anvers, ed. par G. DE LonaÉ, Brüssel 1871, Bd. II, S. 376; Titel XLIX: van den vrij jaermercten, art. 1 und 3).

26 Anders Huvelin, Essai historique, a. a. O., S. 264: „der letzte Montag des August", also vom 25 . bis 31 . August.

27 Hansisches UB., Bd. 8, nr. 94.

${ }^{28}$ Die Tabelle enthält alle Angaben, die in den Bänden 5; 7,1.; 8 des Hansischen UB. zu finden sind; vgl. noch ebenda, Bd. 7,1, nr. 342 u. 749, sowie Bd. 8, nr. 1031. 


\begin{tabular}{lrll}
\hline & \multicolumn{3}{l}{ Bamysse } \\
\hline $\begin{array}{l}\text { HUB } 7,1 \\
\text { nr. 127 }\end{array}$ & 1435 & 28. August-24. September & 20. September \\
HUB 8 & & & \\
nr. 94 & 1451 & 29. August-25. September & 17. September \\
422 & 1455 & 24. August-20. September & 26. September \\
968 & 1460 & 24. August-20. September & 22. September \\
1190 & 1462 & 29. August-25. September & 29. September
\end{tabular}

In den Veckinchusenschen Handelsbüchern werden die Antwerpener Messen mehrfach genannt. Diese Angaben widerlegen einige in der Literatur verbreitete Behauptungen. Entgegen der Meinung, daß die Antwerpener Messen erst im Jahre $1415^{29}$ oder sogar am Ende des ersten Viertels des 15. Jh. ${ }^{30}$ begründet wurden, wird die Bavomesse im Handelsbuch schon in einer Eintragung des Jahres 1404 (Buch Af 1, fol. 38 r.) erwähnt. Die Stelle enthält auch die älteste Erwähnung einer Zahlung auf der Antwerpener Messe, so daß dieser Beleg um ein Jahrzehnt älter ist als der bisher als ältester bekannte. ${ }^{31}$

Alle Datenangaben über die Messen werden in folgender Weise zusammengefaßt: 1. Alphabetische Ordnung der Messen, 2. Jahreszyklus der Messen und innere Zeiteinteilung, 3. Tabelle der Messedaten für die Jahre 1400 bis 1425 (am Schluß des Buches).

\section{Messen in alphabetischer Ordnung}

IX. Antwerpen, Herbst-(Bavo-)Messe

IV. Antwerpen, Pfingstmesse

II. Brügge

VI. St. Denis (Lendit)

V. Kortryk (Courtrai)

VI. Lendit s. St. Denis

VIII. Lille (Ryssel)

X. Messine (Messen)

VII. Thourout

I. Ypern

III. Ypern, Tempelmarkt

(Die römischen Ziffern bedeuten die Stelle der Messe im Jahreszyklus.)

29 Hoveurn, Essai historique, S. 254.

30 Cambridge Economic History, Bd. 3, S. 150.

31 Van Houtte, La Foire, a. a. O., S. 199. 
Jahreszyklus der Messen und innere Zeiteinteilung

I. Ypern ${ }^{32}$ (vgl. Tabelle 3)

Anfang: Aschermittwoch

Schautage: Mittwoch-Freitag nach Reminiscere (Quelle 1295)

Ende: Donnerstag der Woche Letare, der der 30. Tag nach Aschermittwoch ist.

II. Brügge ${ }^{33}$ ( $\nabla g l$. Tabelle 3)

Anfang: Montag nach Quasimodo

Schautage: Montag-Mittwoch nach Jubilate

Ende: Dienstag vor Himmelfahrt

III. Ypern (Tempelmarkt) ${ }^{34}$ (vgl. Tabelle 3)

Anfang: Sonntag vor Himmelfahrt (Quelle 1427)

Ende: Samstag nach Himmelfahrt

IV. Antwerpen (Pfingstmesse) (vgl. Tabelle 3)

Anfang: 15 Tage vor Pfingsten

Schautage: Montag-Mittwoch der Pfingstwoche ${ }^{35}$

Ende: 1. Sonntag nach Trinitatis ${ }^{36}$

V. Kortryk (Courtrai) ${ }^{37}$ (vgl. Tabelle 3)

Anfang: 8 Tage vor Pfingsten

Schautage: Montag-Mittwoch nach der Pfingstwoche (Quelle 1295)

Ende: 15 Tage nach Pfingsten

VI. St. Denis (Lendit) (vgl. Tabelle 3)

Anfang: Zweiter Mittwoch im Juni

Ende: 23. Juni ${ }^{38}$

VII. Thourout (Torrolt) ${ }^{39}$

Anfang: 24. Juni

32 Des Marez, a. a. O., S. 81, 79, 86. Es ist aber zu beachten, daß in einem Schaltjahr, wenn Ostern auf den 15. April fällt, der Aschermittwoch - also der Anfang der Messe - auf den 29. Februar fallen muß.

$33 \mathrm{Vgl}$. oben.

34 Des Marez, a. a. O., S. 81 mit Anm. 3.

35 Über die Schautage habe ich in der Literatur nichts finden können. Ich gebe diese unsichere Datierung an in der Annahme, daß die innere Zeiteinteilung der Antwerpener Messen der der flandrischen ähnlich war.

36 Huvelin gibt die Dauer der Messe mit 29 Tagen an.

37 Über die Kortryker Messe haben wir nur eine einzige Angabe. Nach der Keure von 1324 beginnt die Messe eine Woche vor Pfingsten und dauert bis 15 Tage nach Pfingsten (,une franchisse chascun an commence huits jours avant la Pentecoste, si dure quinze jours après", in: L. WARNKOENIG, Flandrische Staats- u. Rechtsgeschichte, Bd. 2,2, 1837, S. 139-145). Es ist nicht deutlich, ob hier der Pfingstsonntag oder die Pfingstwoche gemeint ist. Im ersten Falle würde die Messe im ganzen drei Wochen, im zweiten vier Wochen dauern, wie die anderen großen flandrischen Messen.

38 Cambridge Economic History, Bd. 3, S. 25.

39 Des Marez, a. a. O., S. 80, 84, 79. 
Schautage: 8.-10. Juli (Quelle 1295)

Ende: 24. Juli

VIII. Lille (Ryssel) 40

Anfang: 16. August

Schautage: 30. August-1. September (Quelle 1295)

Ende: 15. September

IX. Antwerpen, Herbstmesse

(Bavomesse, Bamysse, Michaelismesse ${ }^{41}$ ) (vgl. Tabelle 3)

Anfang: Zweiter Sonntag nach Mitte August (23.-29. August) ${ }^{42}$ oder letzter Montag im August (24.-31. August) 43

Schautage: 6.-12. September oder 7.-14. September

Ende: 19.-25. September oder 22.-29. September

X. Messine (Messen)

Anfang: 1. Oktober

Schautage: 13.-15. Oktober

Ende: 1 . November ${ }^{44}$

40 Ebenda, S. 83 und 84.

41 So in den hansischen Quellen genannt; vgl. „umtrent Banysse" (Hanserecesse, Bd. 5, nr. 205, S. 139).

42 Van Houtte, a. a. O., S. 189.

${ }^{43}$ HUVELIN, a. a. O., S. 264. Im großen und ganzen ist der Unterschied unbedeutend; für einige Jahre jedoch gehen die Daten - wenn die eine oder die andere Datierung angenommen wird - um acht Tage auseinander.

44 Des Marez, a. a. O., S. 82 und 79, Anm. 1. 